\title{
Nasonia Parasitic Wasps Escape from Haller's Rule by Diphasic, Partially Isometric Brain-Body Size Scaling and Selective Neuropil Adaptations
}

\author{
Jitte Groothuis Hans M. Smid \\ Laboratory of Entomology, Wageningen University, Wageningen, The Netherlands
}

\section{Keywords}

Haller's rule · Brain · Insect - Nasonia vitripennis - Parasitic wasp · Confocal laser scanning microscopy Plasticity .

Neuropil $\cdot$ Mushroom body

\begin{abstract}
Haller's rule states that brains scale allometrically with body size in all animals, meaning that relative brain size increases with decreasing body size. This rule applies both on interand intraspecific comparisons. Only 1 species, the extremely small parasitic wasp Trichogramma evanescens, is known as an exception and shows an isometric brain-body size relation in an intraspecific comparison between differently sized individuals. Here, we investigated if such an isometric brainbody size relationship also occurs in an intraspecific comparison with a slightly larger parasitic wasp, Nasonia vitripennis, a species that may vary 10 -fold in body weight upon differences in levels of scramble competition during larval development. We show that Nasonia exhibits diphasic brainbody size scaling: larger wasps scale allometrically, following Haller's rule, whereas the smallest wasps show isometric scaling. Brains of smaller wasps are, therefore, smaller than expected and we hypothesized that this may lead to adaptations in brain architecture. Volumetric analysis of neuropil
\end{abstract}

\begin{tabular}{ll}
\hline KARGER & $\begin{array}{l}\text { ( } 2017 \text { The Author(s) } \\
\text { Published by S. Karger AG, Basel }\end{array}$ \\
$\begin{array}{l}\text { E-Mail karger@karger.com } \\
\text { www.karger.com/bbe }\end{array}$ & $\begin{array}{l}\text { This article is licensed under the Creative Commons Attribution- } \\
\text { NonCommercial-NoDerivatives 4.0 International License (CC BY- } \\
\text { NC-ND) (http://www.karger.com/Services/OpenAccessLicense). } \\
\text { Usage and distribution for commercial purposes as well as any dis- } \\
\text { tribution of modified material requires written permission. }\end{array}$
\end{tabular}

composition revealed that wasps of different sizes differed in relative volume of multiple neuropils. The optic lobes and mushroom bodies in particular were smaller in the smallest wasps. Furthermore, smaller brains had a relatively smaller total neuropil volume and larger cellular rind than large brains. These changes in relative brain size and brain architecture suggest that the energetic constraints on brain tissue outweigh specific cognitive requirements in small Nasonia wasps.

(c) 2017 The Author(s)

Published by S. Karger AG, Basel

\section{Introduction}

Haller's rule states that small animals have relatively larger brains than larger animals, and this has been supported by studies throughout the animal kingdom [Rensch, 1948]. One explanation for this relationship between body size and brain size is that smaller individuals require the same cognitive abilities as larger ones, and hence cannot scale their brain size at the same rate as their body size. This brain-body size relationship is a negative allometry, which can be visualized by a logarithmically transformed plot, where $\ln$ (brain size) scales linearly to $\ln$ (body size) with a slope, or allometric coefficient " $b$ " 


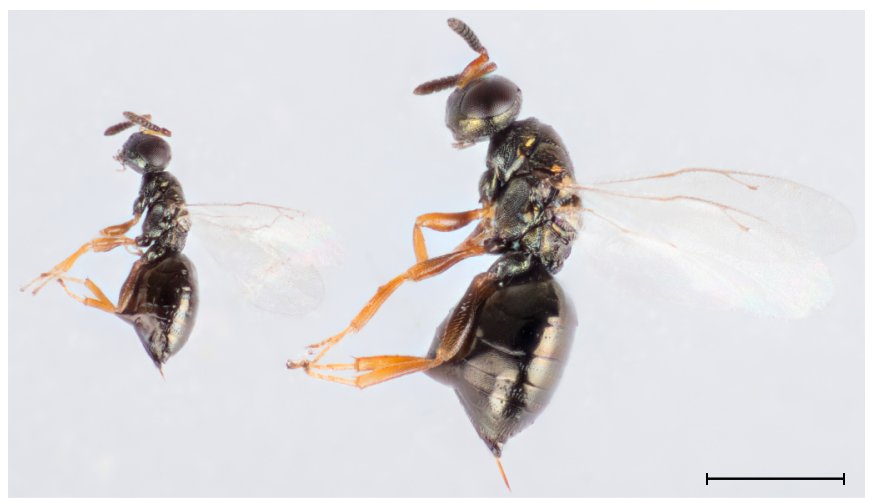

Fig. 1. Two genetically identical individuals of the isogenic N. vitripennis AsymCx line, subjected to different rearing conditions. The body (thorax + abdomen) length of the smallest wasp is 1,375 $\mu \mathrm{m}$ and its head width is $469 \mu \mathrm{m}$. The largest wasp measures 2,438 and $773 \mu \mathrm{m}$, respectively. Scale bar, $1 \mathrm{~mm}$.

that is smaller than 1. An isometric relationship would have a slope that is not different from 1 . The negative allometry between brain size and body size holds true for inter- and intraspecies comparisons [Pagel and Harvey, 1988], but intraspecific comparisons yield lower slope values (indicating stronger allometry) than interspecific comparisons at higher taxonomic levels [Pagel and Harvey, 1988, 1989; Wehner et al., 2007]. Diphasic intraspecific allometry has been described in ants, where small ants had a higher slope value than larger ants [Seid et al., 2011].

The smallest animal with a described intraspecific brain-body size relation is the wasp Trichogramma evanescens, an egg parasitoid with a body length of 0.3-0.9 $\mathrm{mm}$. Remarkably, brain and body volume scaling shows isometry in this species [van der Woude et al., 2013]. To our knowledge, T. evanescens is the only animal described that exhibits this isometry, thereby "escaping" Haller's rule. This may be because their brain volume comprises on average $8 \%$ of the body volume [van der Woude et al., 2013], which likely entails high metabolic costs that strongly constrain brain development in smaller individuals. Thus, isometry in Trichogramma wasps, and diphasic brainbody size scaling in small ants (with a higher allometric coefficient for the smallest individuals) may both be a consequence of miniaturized body size and the resulting high metabolic costs of their relatively large brain [Chittka and Niven, 2009; Eberhard and Wcislo, 2011].

The development of smaller brains, especially in cases where brains develop to be smaller than expected from
Haller's rule, may be accommodated by a reduction in complexity, such as disproportionate scaling of neuropils. Such compensatory changes may be seen as the outcome of a trade-off between the energy requirements of neural tissue, which become larger in smaller individuals as relative brain size increases, and the requirement to maintain cognitive functions in the smallest wasps determined by their ecological relevance [Riveros and Gronenberg, 2010; Muscedere and Traniello, 2012; Stöckl et al., 2016; van der Woude and Smid, 2017a]. Examples of absolute brain size influencing neuropil composition include Drosophila melanogaster, for which the optic lobe size is relatively smaller in smaller individuals [Lanet et al., 2013]. A decrease in relative optic lobe volume in smaller brains is also observed in a comparison of 13 paper wasp species [O'Donnell et al., 2013]. In T. evanescens, the relative volume of antennal lobe glomeruli is smaller in the smallest wasps, although their number is constant [van der Woude and Smid, 2016], whereas relative antennal lobe volume is not influenced by brain or body size in much larger species like bumblebees and honeybees [Mares et al., 2005; Gronenberg and Couvillon, 2010].

Here, we investigated whether isometric brain-body size scaling and disproportionate neuropil scaling occurs in a parasitic wasp species with a less extreme level of body miniaturization, the jewel wasp Nasonia vitripennis Walker, 1836 (Hymenoptera: Pteromalidae). Nasonia vitripennis are small parasitic wasps, generally $1.5-2.5 \mathrm{~mm}$ in length [Werren and Loehlin, 2009]. They are ectoparasitoids of various fly species and the size of emerging wasps depends on the size and quality of the host [Hoedjes et al., 2014] and the number of eggs laid inside a host puparium [Charnov and Skinner, 1984; Sykes et al., 2007]. Increasing the number of eggs per puparium increases scramble competition between the developing larvae, which can result in a 10-fold reduction in body dry weight (Fig. 1). Comparable body size differences may also be obtained in other species or lines, such as $N$. giraulti or the outbred N. vitripennis HVRx line [van de Zande et al., 2014], as shown in online supplementary material S1 (see www.karger.com/doi/10.1159/000480421 for all online suppl. material). In nature, host quality and abundance likely varies profoundly, as well as the number of competing Nasonia females laying eggs.

The strong plasticity of body size in N. vitripennis permits the study of Haller's rule in this wasp species as a comparison to the minute Trichogramma wasp [van der Woude et al., 2013]. Moreover, by using an isogenic $\mathrm{Na}$ sonia strain we exclude an effect of genetic variation on brain-body scaling. Assuming that the isometric brain- 
body size relation in T. evanescens is related to its extremely small size, we expected to find an allometric, but possibly diphasic, brain-body size relation in the bigger wasp N. vitripennis.

To investigate the effect of size variation on brain complexity in $N$. vitripennis, we compared relative volumes of specific neuropils of the largest wasps with the smallest wasps. We hypothesized that neuropil regions in the brains of differently sized $N$. vitripennis wasps show different degrees of scaling. Based on the knowledge that olfaction is of greater ecological relevance than vision for host location in N. vitripennis [Whiting, 1967] and the examples mentioned above, we expected to find larger effects of size plasticity in the optic lobes than the other neuropil regions, such as the antennal lobe.

\section{Material and Methods}

\section{Insect Rearing}

Nasonia vitripennis Walker, 1836 (Hymenoptera: Pteromalidae) wasps of the isogenic AsymCx strain were kept as described previously [Hoedjes et al., 2012]. In short, for a rearing of regularsized wasps, wasps were reared on Calliphora vomitoria pupae (Kreikamp BV, Hoevelaken, The Netherlands) using 10 mated females to 20 fly pupae at $25 \pm 1^{\circ} \mathrm{C}$ and 16:8 (L:D) photoperiod. To induce more size variation, wasps were reared as above, except at 50 female wasps to 5 fly pupae. The wasps could oviposit for $24 \mathrm{~h}$. After emergence, all wasps were kept overnight in vials with $\mathrm{H}_{2} \mathrm{O}$ and honey prior to experiments. Only female wasps were considered for this study. Only 1- to 2-day-old naive wasps were used to prevent any confounding factors and minimize the influence of ontogenetic plasticity.

\section{Head and Body Measurements}

Measuring brain volume as wet tissue weight in $N$. vitripennis was expected to generate large errors because of the small size and fragile nature of the brains [Haverkamp and Smid, 2014]. Previous studies avoided this problem and investigated the brain volume by using confocal laser scanning microscopy and subsequent $3 \mathrm{D}$ volume reconstruction through the intact head capsule, which was either transparent by nature [van der Woude et al., 2013] or after clearing [Smolla et al., 2014]. The latter procedure did not yield satisfactory results to unveil the brain in the black head capsule of $N$. vitripennis because of strong deformation of the brain tissue (data not shown). However, van der Woude et al. [2013] showed a strong correlation between head and brain volume in T. evanescens. In this species, the head capsule is tightly connected to the brain. Such a correlation could be lower in larger insects with more glands or musculature (such as ant major workers or Vespid wasps, where head capsule volume is used as a proxy for body size [Bulova et al., 2016; O'Donnell and Bulova, 2017]). In online supplementary material S4 we show that this is not the case for N. vitripennis. Accordingly, we decided to use head capsule volume and body dry weight, respectively, as proxies for brain and body volume. These proxies allowed analysis of many wasps at a high ac- curacy, yielding a robust data set while maintaining the ability to correlate individual head and body measurements.

Nasonia vitripennis individuals reared with induced size plasticity have a larger variation in size than those reared under standard conditions. Therefore, to obtain a complete representation of size variation in this species, 60 randomly selected wasps from this population were analyzed, in addition to 24 wasps reared under standard conditions. Following sedation on a $\mathrm{CO}_{2}$ pad (Genesee Scientific), body (thorax + abdomen) length, hind tibia length, and head width were determined using an ocular micrometer. Wasps were decapitated with sharp tweezers (Dumont No. 5; Sigma) and the bodies were transferred to a 96-well plate (Greiner Bio-One), while the heads were processed as described below. The bodies were dried at $65^{\circ} \mathrm{C}$ for $1 \mathrm{~h}$ and subsequently weighed on a Sartorius $\mathrm{CP} 2 \mathrm{P}$ microbalance. Weighing was performed twice and the average was used in the analyses. There was a very low measurement error for these measurements, with a high correlation between the first and second weighing (Pearson $r=0.9998$ ).

To measure the head capsule volume of these decapitated wasps, an adaptation of previously used methods [Smolla et al., 2014; Werren et al., 2016] was used. After removal of the antennae, the heads were placed in a 96-well plate and fixed for $24 \mathrm{~h}$ at room temperature (RT) in $4 \%$ formaldehyde in $0.1 \mathrm{M}$ phosphate buffer (pH 7.2), freshly prepared from paraformaldehyde. After fixation, the heads were washed for $24 \mathrm{~h}$ in phosphate-buffered saline (PBS; Oxoid, Dulbecco "A" tablets) at RT, followed by incubation in 30\% $\mathrm{H}_{2} \mathrm{O}_{2}$ (Sigma) for 7-10 days at RT until the cuticle was an opaque white. The heads were then washed 4 times over $2 \mathrm{~h}$ in PBS at RT and subsequently dehydrated through ethanol solutions of increasing concentration $(30,50,70,80,90,96$, and $100 \%, 2$ min each) followed by an incubation in 50/50 ethanol/xylene, and $100 \%$ xylene for clearing. The heads were mounted in DPX mounting medium (Sigma) between 2 No. 1 cover slips, separated by a custom spacer ( 2 stacked strips of adhesive tape, Henzo, Roermond, The Netherlands).

For confocal laser scanning microscopy, preparations were placed in a Cobb slide [Cobb, 1917] (purchased from the Laboratory of Nematology, Wageningen University and Research). A Zeiss LSM510 microscope equipped with a $10 \times$ objective (Plan-Apochromat $10 \times / 0.45$ ) with a $512 \times 512$-pixel resolution was used for image acquisition. Excitation was induced with the 488-nm line of an Argon laser. The emission of autofluorescence was captured with 505$550 \mathrm{~nm} \mathrm{BP}$ and $560 \mathrm{~nm}$ LP filters. Heads were scanned with a step size of $5 \mu \mathrm{m}$ from both directions. To compensate for axial scaling due to refraction index mismatch [Bucher et al., 2000] between air and DPX, the voxel depth was scaled with a correction factor of 1.52. The final voxel calibration was $1.7995 \times 1.7995 \times 7.6 \mu \mathrm{m}$.

Anterior and posterior Z-stacks were flipped and rotated to the same orientation in FIJI [Schindelin et al., 2012] and fused with the Pairwise Stitching plugin [Preibisch et al., 2009]. The head capsule was segmented every $4-5$ slices using the segmentation editor in Amira 5.4.2 (Visage Imaging) with the Threshold and Brush tools. Interpolation was used to label the entire head; to ensure correct interpolation, all slices were inspected and corrected, if necessary. The head capsule volume was calculated with the MaterialStatistics module.

\section{Neuropil Stainings}

To compare the neuropil distribution in $N$. vitripennis wasps of different sizes, 2 extreme groups of new wasps were analyzed. We 
selected small wasps with a head width under $500 \mu \mathrm{m}$, and large wasps with heads over $750 \mu \mathrm{m}$ wide. As the brains were pooled per size class during staining procedures, no further individual measurements were performed on these wasps.

Cold-sedated wasps were decapitated in ice-cold PBS. Using fine sharpened tweezers, the cuticle was removed and brains dissected. Dissected brains were kept in freshly prepared ice-cold $4 \%$ formaldehyde. After obtaining several brains, they were placed in fixative solution at RT and fixed for $2.5 \mathrm{~h}$. After rinsing 6 times for $5 \mathrm{~min}$ in PBS, the brains were treated in $5 \mathrm{mg} / \mathrm{mL}$ collagenase (Sig$\mathrm{ma}$ ) in PBS for $1 \mathrm{~h}$ at RT, improving the permeability of the tissue. Subsequently, the brains were rinsed 4 times for 5 min in PBS containing $0.5 \%$ Triton-X100 (PBS-T) and preincubated in 10\% normal goat serum (NGS; Dako, Glostrup, Denmark) in PBS-T (PBST-NGS) for $1 \mathrm{~h}$ at RT. The brains were then incubated overnight at RT in the primary antibody (1:250 dilution of mouse anti-bruchpilot; concentrate, DSHB hybridoma product nc82 [Wagh et al., 2006], in PBS-T-NGS). Brains were rinsed 6 times for $20 \mathrm{~min}$ in PBS-T and incubated in 1:200 AlexaFluor ${ }^{\circledR} 488$-conjugated goat anti-mouse (Invitrogen) and 1:500 propidium iodide (Sigma-Aldrich) for $4 \mathrm{~h}$. Brains were rinsed 4 times for $30 \mathrm{~min}$ in PBS-T and 2 times for $10 \mathrm{~min}$ in PBS. To avoid tissue shrinkage, which could lead to a confounding distortion of relative volumes, the brains were not dehydrated and cleared. Instead, they were mounted in aqueous VectaShield (Vector Laboratories) between two $24 \times 24$ $\mathrm{mm}$ coverslips with custom spacers and placed in a Cobb slide for imaging. Although the lack of clearing slightly diminished image quality, this did not outweigh the advantage we gained by comparing in situ volumes and posed no problem in segmenting neuropils.

Preparations were scanned with a Zeiss LSM510 microscope equipped with a $25 \times$ oil immersion objective (Plan-Neofluar $25 \times / 0.8)$. AlexaFluor ${ }^{\circledR} 488$ staining was imaged using the $488-\mathrm{nm}$ line of an Argon laser with a 505- to 550-nm BP filter; the propidium iodide nuclear counterstain was imaged using the same excitation wavelength but with a 560-nm LP filter. To accommodate the entire brain, 10-20\% overlapping side-by-side Z-stacks were scanned from both sides of the preparation at 1,024 $\times 1,024$ pixels with a digital magnification of 0.8 and a step size of $2 \mu \mathrm{m}$. To compensate for axial scaling due to refraction index mismatch [Bucher et al., 2000] between immersion oil and VectaShield, a correction of 0.9505 was applied to the voxel depth. The final voxel calibration was $0.4498844 \times 0.4498844 \times 1.901 \mu \mathrm{m}$. After image acquisition, the entire brain was reconstructed in FIJI as described above for the whole head mounts.

Fig. 2. Overview of the analyzed neuropils. a Slices through a single $N$. vitripennis brain from the large population, fluorescently labelled with nc82 (green) and propidium iodide (magenta). The slice depth is indicated in the top right corner of each image. Slice orientation in $\mathbf{a}$ and $\mathbf{b}$ as in $\mathbf{c}$, and refers to the body axis [Haverkamp and Smid 2014; Ito et al., 2014]. b Corresponding labels of the cell body rind and segmented neuropils: optic lobes, i.e., lobula (LO), medulla (ME), and lamina (LA); mushroom body, i.e., calyx (CA), pedunculus (PED), vertical lobe (VL, not visible in $\mathbf{b}$ ), and medial lobe (ML); central complex, i.e., fan-shaped body (FB), ellipsoid

\section{Neuropil Analysis}

Complete image stacks were imported in Amira and the nc82 channel was used in the Segmentation Editor to assign voxels to 13 distinct neuropil regions. Standardized nomenclature [Ito et al., 2014] was used where possible. We included optic lobes (i.e., lobula, medulla, lamina), anterior optic tubercle, lateral horn, antennal lobe, mushroom body (i.e., calyx and ventral mushroom body [i.e., pedunculus, vertical, medial lobes]), central complex (i.e., fan-shaped body, ellipsoid body, noduli, protocerebral bridge), and rest of neuropils. When possible, the cell body rind (the outermost layer of the arthropod brain, containing all neuronal cell bodies) was also segmented. For an overview, see Figure 2. Labels were assigned every 1-5 slices and completed with the Interpolation option. All slices were inspected and interpolation errors corrected when necessary. The MaterialStatistics module was used to compute the absolute neuropil volumes. Volumetric data were imported in MS Excel and used to calculate relative volumes by dividing a neuropil volume by the total neuropil volume of that brain. For the confocal slices selected as illustration in Figure 2, the image contrast was optimized for viewing in FIJI. The surface models of individual brains selected for illustration were generated using the Amira SurfaceGen module.

\section{Statistics}

There is considerable discussion on the use of different regression methods in the study of allometry [Smith, 2009; Voje et al., 2014; Kilmer and Rodríguez, 2017]. A particular topic of interest is the choice between ordinary least-squares (OLS) and (standardized) major axis (SMA) regression, and what is actually described by slopes determined by these methods. In this work, we followed the advice of Kilmer and Rodríguez [2017] and used OLS regression to describe the relationship between the body size and brain size of $N$. vitripennis.

Piecewise regression analyses were performed as described by Crawley [2007]. This method compares a linear regression model to a model that consists of 2 parts with distinct slopes. The break point where the slope changes was found by developing several regression models, each using a break point at a different value based on the unique weights in the dataset. The model with the lowest residual standard error was selected as the 2-slope model that best described the data. One-way analysis of variance (ANOVA) tests were used to compare the 1-slope and 2-slope regression models, and test which one provided a better fit. Similar analyses were used to test if a model with 3 slopes explained the data better than a 2-slope model.

body $(\mathrm{EB})$, protocerebral bridge $(\mathrm{PB})$, and noduli $(\mathrm{NO})$; lateral horn ( $\mathrm{LH})$; antennal lobe (AL), and rest of neuropil (RoN). Scale bar, $200 \mu \mathrm{m}$ (representative for $\mathbf{a}$ and b). c, d Anterior surface renderings based on the large brain shown in $\mathbf{b}(\mathbf{c})$, and a small brain (d). The cell body rind and RoN are transparent. e, f Posterior views of the same brains as in $\mathbf{c}$ and $\mathbf{d}$, respectively. Labels in $\mathbf{c}-\mathbf{f}$ as in $\mathbf{b}$, with addition of the anterior optic tubercle (AOTu, not visible in b). These individual brains differ 2.1-fold in total volume, but the large brain is $61.8 \%$ neuropil, whereas the small brain is $47.1 \%$ neuropil. Scale bar, $200 \mu \mathrm{m}$ (representative for c-f).

(For figure see next page.) 
We used OLS regression models to estimate the coefficients of the 1-slope and 2-slope relationships between brain size and body size. To test for isometry, we determined whether the slope was significantly different from 1 using the smatr package [Warton et al., 2012]. All regression analyses were performed in $\mathrm{R}$ version 3.3.2.

To compare the volumes of brain compartments we used a multivariate linear model with the absolute or relative volume of the various neuropils as dependent variables and the size class (small or large) as the fixed factor. This analysis was performed in $\mathrm{R}$ version 3.3.2. As we tested many variables (neuropils) at once, we corrected $p$ values of pairwise comparisons by means of the Holm-Bonferroni method for multiple comparisons [Holm, 1979]. For supercategories [Ito et al., 2014] we used $m=$ 7 , and for separate neuropils this was $m=13$. This correction was performed in MS Excel. An a level of 0.05 was used for all analyses.


c
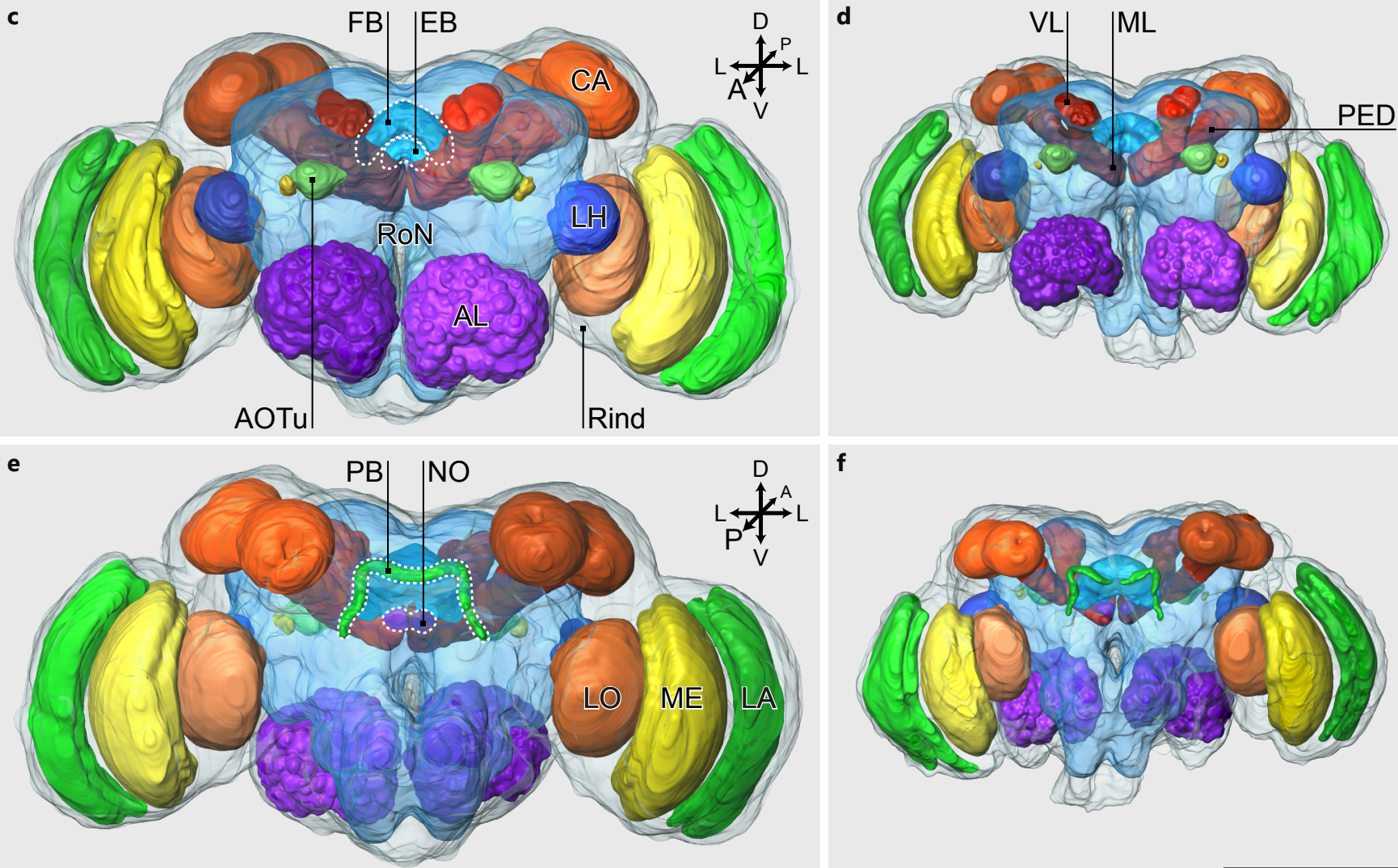

f

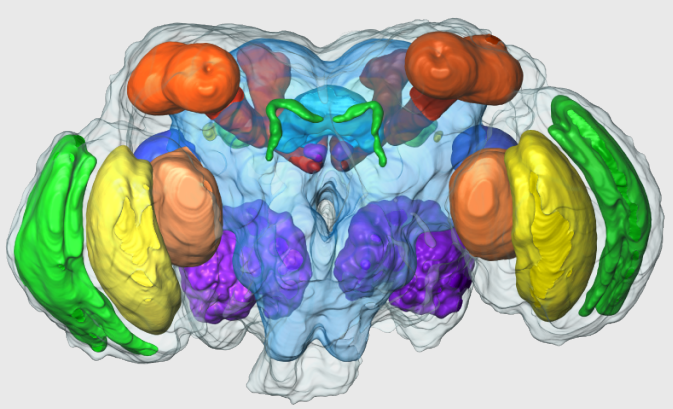




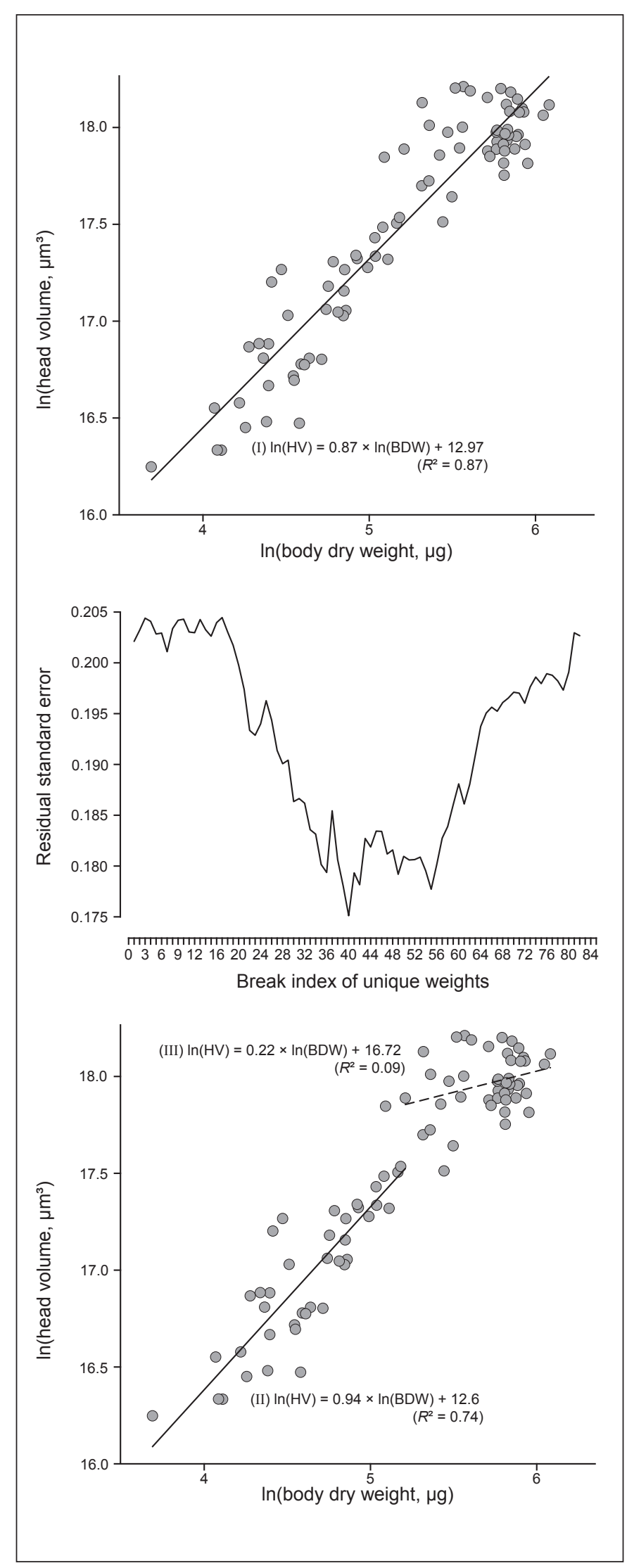

Data Accessibility

The dataset supporting brain-body size scaling is part of online supplementary Table S2. Raw and analyzed volumetric data for all brains are part of online supplementary Table S3.

\section{Results}

Intraspecific Brain-Body Size Scaling in Isogenic

$\mathrm{N}$. vitripennis

Body dry weights ranged from 40 to $438 \mu \mathrm{g}$, corresponding to body lengths of $1,200-2,420 \mu \mathrm{m}$. Head volumes ranged from $11.4 \times 10^{6}$ to $81.1 \times 10^{6} \mu \mathrm{m}^{3}$, corresponding to head widths of $414.5-803.1 \mu \mathrm{m}$. These results (Fig. 3a) indicate that, under high scramble competition, $N$. vitripennis females have an adult (dry) body weight range spanning at least one order of magnitude. An overview of all measurements is given in online supplementary Table S2.

OLS regression on the natural logarithms of body dry weight and head capsule volume revealed a negatively allometric brain-body size relationship. The allometric coefficient $(0.87,95 \%$ CI $0.80,0.94)$ was significantly different from $1(p=0.0006)$.

(I) $\begin{aligned} \ln (\text { head volume })= & 0.87 \times \ln (\text { body dry weight }) \\ & +12.97\left(R^{2}=0.87\right)\end{aligned}$

A 2-segment piecewise regression model with a break point at $183 \mu \mathrm{g}\left(R^{2}=0.91\right.$; see Figure $3 \mathrm{~b}$ for the break point analysis) explained the data better than a linear regression model (ANOVA, $F_{2,80}=14.618, p<0.001$ ) and showed a significant interaction between body dry weight and wasp size group $(p<0.0001)$. A separate OLS regression on small and large $N$. vitripennis wasps resulted in the relationships described in formulas II and III, respectively. The allometric coefficient of the small wasp regression $(0.94,95 \%$ CI $0.76,1.13)$ was not significantly different from $1(p=0.54)$. The allometric coefficient of the large wasp regression $(0.22,95 \%$ CI $0.00,0.44)$ was different from $1(p<0.0001)$ but not from $0(p=0.053)$. This

Fig. 3. Brain-body size scaling in N. vitripennis depicted by logarithmic plots of body dry weight and head capsule volume. a The result of single-slope OLS regression (formula I). b Residual standard error of multiple regression models ( $y$ axis), plotted for all unique body dry weights (index on $x$ axis). The lowest error is found at index 40, corresponding to $183 \mu \mathrm{g}$. The second lowest error is at index 52, corresponding to $302 \mu \mathrm{g}$. c Diphasic allometry: solid line, OLS regression on wasps under $183 \mu \mathrm{g}$ (formula II); dashed line, OLS regression on wasps over $183 \mu \mathrm{g}$ (formula III). 
indicates that wasps with a body dry weight lower than $183 \mu \mathrm{g}$ show isometric brain-body size scaling, whereas larger wasps show negative allometry, or have an invariable brain size. We noted that the fit of the allometric line in the larger wasps is not optimal $\left(R^{2}=0.09\right)$, which may be caused in part by insufficient variation of the larger wasps on the ln-transformed $x$ axis.

$$
\begin{aligned}
\text { (II) } \ln (\text { head volume })= & 0.94 \times \ln (\text { body dry weight }) \\
& +12.6\left(R^{2}=0.74\right) \\
\text { (III) } \ln (\text { head volume })= & 0.22 \times \ln (\text { body dry weight }) \\
& +16.72\left(R^{2}=0.09\right)
\end{aligned}
$$

Three-segment piecewise regression was not found to be an improvement over the model with 2 slopes (ANOVA, $F_{2,78}=0.6921, p=0.50$ ); therefore, no 3-segment OLS regression was performed. A 2 -segment model with the breakpoint at the second lowest residual standard error (at $302 \mu \mathrm{g}$ ) was also no better than the 183- $\mu \mathrm{g}$ model. In addition, we checked if other (nonlinear) functions in log space could better describe the brain-body size scaling. A power law, as well as multiple degrees of polynomial functions, did not have a better fit than the 2-segment piecewise regression presented above.

\section{Total Brain Composition}

Small wasps with a head width under $500 \mu \mathrm{m}$ and large wasps with heads more than $750 \mu \mathrm{m}$ wide had an average 2.3 -fold difference in absolute total brain volume (i.e., neuropil with cell body rind; $F_{1,22}=459.32, p<0.001$; Fig. 4). Due to its fragility, the cell body rind was too damaged for segmentation in 4 large brains. Data for the total brain volume of large brains are therefore reported for $n=13$, whereas neuropil volumes of these brains are reported with $n=17$. The largest $N$. vitripennis wasps ( $n=$ 13) measured $30.4 \pm 0.59 \times 10^{6} \mu \mathrm{m}^{3}$ (mean $\pm \mathrm{SE}$ ) in total brain volume, consisting of $60.45 \pm 0.74 \%(18.1 \pm 0.33 \times$ $\left.10^{6} \mu \mathrm{m}^{3}\right)$ neuropil, with $39.55 \pm 0.74 \%\left(12.1 \pm 0.37 \times 10^{6}\right.$ $\mu \mathrm{m}^{3}$ ) dedicated to the rind. Total brain in the smallest wasps $(n=11)$ was $13.3 \pm 0.52 \times 10^{6} \mu \mathrm{m}^{3}$, of which 54.52 $\pm 0.86 \%\left(7.2 \pm 0.31 \times 10^{6} \mu^{3}\right)$ was neuropil and $45.48 \pm$ $0.86 \%\left(6.0 \pm 0.27 \times 10^{6} \mu \mathrm{m}^{3}\right)$ rind. In terms of absolute neuropil volume, this is a $151.73 \%$ increase (i.e., approximately 2.5 -fold) in the large wasps compared to the smallest wasps. The differences in relative total neuropil and relative cell body rind are significant, $F_{1,22}=27.72$, $p<$ 0.001 .

\section{Neuropil Scaling}

At the level of neuropil supercategories (Fig. 5), small wasps had a smaller relative volume of the optic lobes $\left(F_{1,26}=40.93\right.$, corrected $\left.p<0.001\right)$ and mushroom bodies

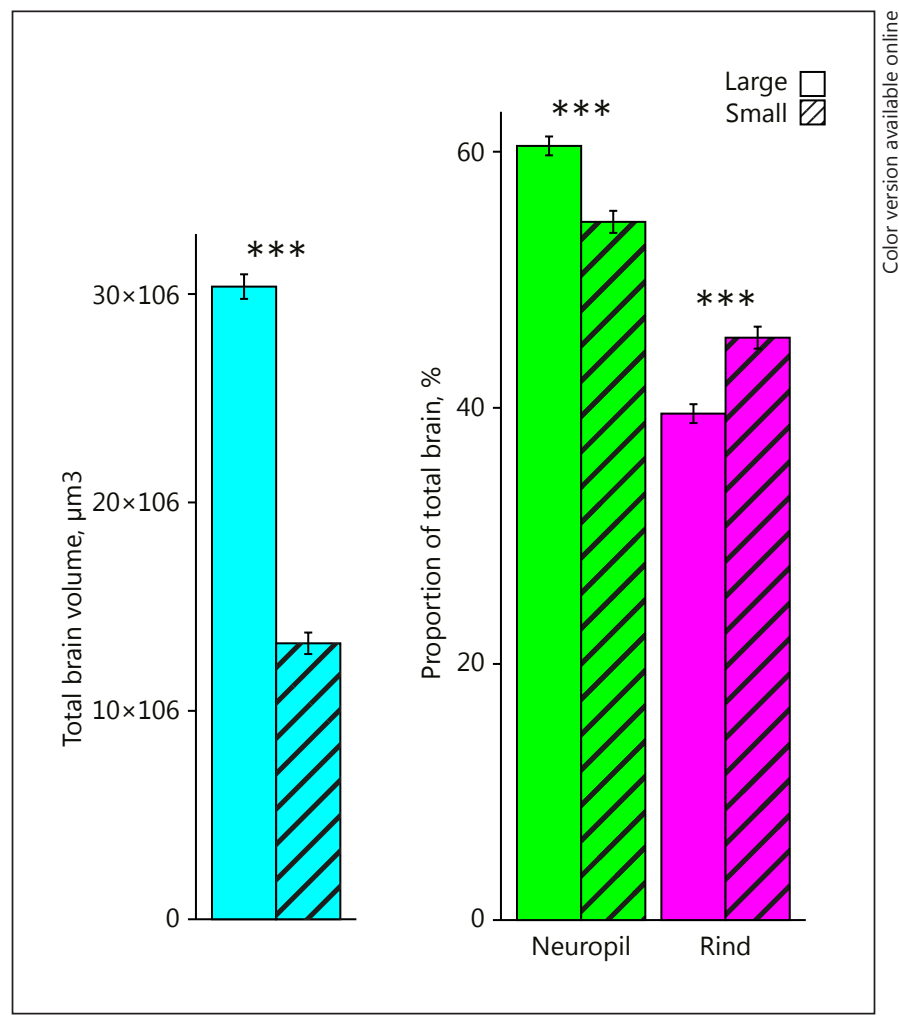

Fig. 4. Total brain volume. a Differences in total brain volume. b The proportion of the brain devoted to neuropil or cell body rind. Bar colors match the colors of neuropil and rind in Figure 2a. Large, $n=13$; small $n=11$. Error bars depict the SE. ${ }^{* * *} p<0.001$.

$\left(F_{1,26}=96.00\right.$, corrected $\left.p<0.001\right)$. By contrast, the relative volume of the central complex $\left(F_{1,26}=25.36\right.$, corrected $p<0.001)$ and rest of the neuropil $\left(F_{1,26}=64.86\right.$, corrected $p<0.001$ ) were larger in small wasps. Complete data are reported in online supplementary Table S3.

Further analysis (Fig. 6) revealed that relative volumes of all neuropils of the optic lobes were significantly smaller in small wasps: the lobula $\left(F_{1,26}=32.24\right.$, corrected $p<$ $0.001)$, medulla $\left(F_{1,26}=30.64\right.$, corrected $\left.p<0.001\right)$, and lamina $\left(F_{1,26}=9.60\right.$, corrected $\left.p=0.03\right)$. Analysis of the mushroom body subunits revealed that the difference shown in Figure 5 was largely due to a smaller relative volume of the calyx in small wasps $\left(F_{1,26}=113.44\right.$, corrected $p<0.001)$ rather than the ventral mushroom body (peduncle and lobes), which showed a smaller and marginally insignificant difference $\left(F_{1,26}=7.73\right.$, corrected $p=0.06)$. The larger relative volume of the central complex in small wasps reported in Figure 5 was attributed to the fan-shaped body and ellipsoid body $\left(F_{1,26}=22.89\right.$, corrected $p<0.001$, and $F_{1,26}=32.83$, corrected $p<0.001$, 
Fig. 5. Relative neuropil volumes at supercategory level in large and small $N$. vitripennis. Large, $n=17$; small, $n=11$. The optic lobes (OL, consisting of LO, ME, and LA), mushroom body (MB, consisting of $\mathrm{CA}, \mathrm{PED}, \mathrm{VL}$, and ML), and central complex (CX, consisting of $\mathrm{FB}, \mathrm{EB}, \mathrm{PB}$, and NO) were segmented as single labels. Further abbreviations and bar colors as in Figure $2 \mathrm{~b}-\mathrm{f}$ and the brain image. Error bars depict SE. Asterisks depict significant differences after Holm-Bonferroni correction $(m=7)$. ${ }^{* * *} p<0.001$.

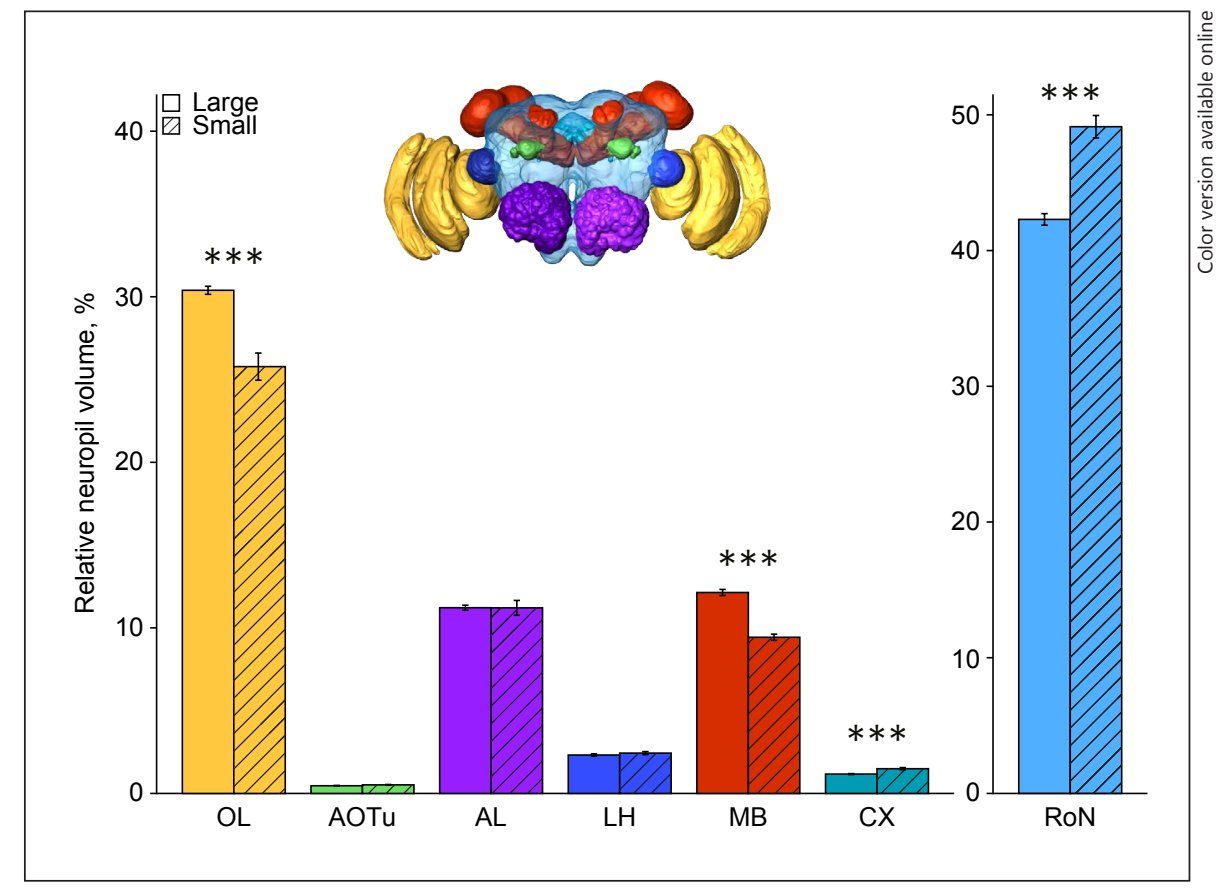

respectively), with no difference in relative volume of the noduli and protocerebral bridge. As in our previous analysis, the relative volume of the rest of the neuropil was larger in small wasps $\left(F_{1,26}=64.86\right.$, corrected $\left.p<0.001\right)$ and no difference was found for the antennal lobe and lateral horn (primary and secondary olfactory neuropils, respectively). Complete data are reported in online supplementary Table S3.

\section{Discussion}

We induced a large degree of body size variation in an isogenic line of the parasitic wasp $N$. vitripennis and found for the first time a diphasic brain-body size relationship where the smallest wasps showed isometry. Though diphasic scaling has been described in insects before [Seid et al., 2011], both phases described showed negative allometry. By contrast, diphasic brain scaling in $N$. vitripennis combines an isometric $(\mathrm{b}=0.94)$ and a negative allometric $(b=0.22)$ phase for smaller and larger wasps, respectively. Our results indicate that $N$. vitripennis may represent a link between isometric scaling in minute wasps [van der Woude et al., 2013] and diphasic allometry in larger insects [Seid et al., 2011]. We present an overview of brain-body size scaling coefficients in several small hymenoptera showing that if body size be- comes smaller, and relative brain size larger, a threshold is reached where negative allometry switches to isometry (Fig. 7).

Although we acknowledge the suboptimal allometric fit $\left(R^{2}=0.09\right)$ for the larger wasps, statistical evidence for the fact that the scaling coefficient for larger wasps differs from 1 is strong. Moreover, we posit that this low fit only strengthens the notion that brain size is more strongly constrained at smaller body sizes than at larger body sizes. As evidenced by the very low allometric coefficient in the large phase and the fact that this value was different from 1 but not from 0 , there is hardly any or no increase in brain size for larger wasps.

Due to the large variation in size in $N$. vitripennis, we expected to find compensatory changes in neuropil composition in extremely small brains. This expectation was strengthened by the isometric brain body size relation that we found in small wasps. We compared volumetric measurements for brains of small and large wasps and indeed found specific neuropils with different relative volumes, whereas others had similar relative volume (Fig. 6).

As expected, smaller individuals have reduced investment in the optic lobes. This correlates with our observation that smaller $N$. vitripennis have fewer and smaller ommatidia (see online suppl. material S5) and with developmental plasticity of the optic lobes observed in smaller 
Fig. 6. Relative neuropil volumes for individual neuropils in large and small $N$. vitripennis. Large, $n=17$; small, $n=11$. The ventral mushroom body (MB-V, consisting of PED, VL, and ML) was segmented as a single label. Further abbreviations and bar colors as in Figure $2 \mathrm{~b}-\mathrm{f}$ and the brain image. Error bars depict SE. Asterisks depict significant differences after HolmBonferroni correction $(m=13){ }^{*} p<0.05$, $* * * p<0.001$.



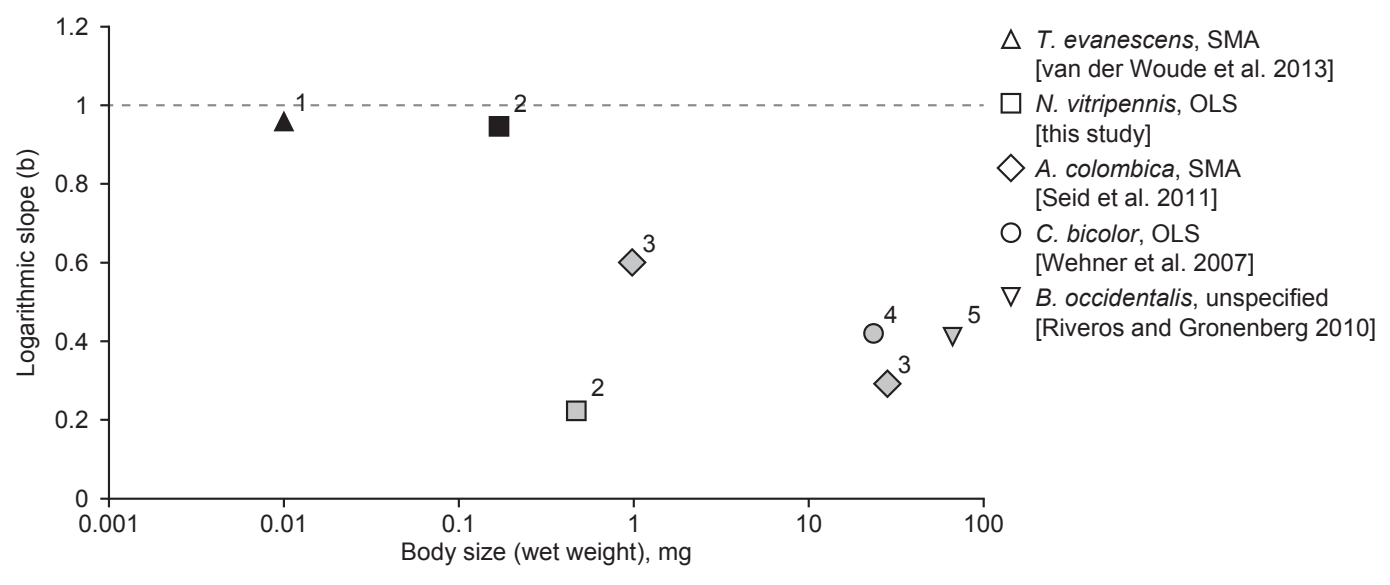

Fig. 7. Brain-body size scaling depends on body size in insects. The 5 species shown here span several orders of magnitude in body size. Brain scaling coefficients are plotted against a common body size measurement: wet weight. For some species, this weight was approximated as indicated. Scaling coefficients are isometric (dotted line at $b=1$ ) in extremely small Hymenoptera (black data points), but allometric when body size increases (gray data points). The slopes reported here were obtained using different regression methods (see Materials and Methods). 1: T. evanescens wasps, wet weight approximated using the density of water. 2: N. vitripennis wasps, wet weight approximated by weighing several sedated wasps (data not shown). Note that the 2 data points correspond to 2 size classes due to the diphasic scaling in this species. SMA regression would result in slightly different slope values $\left(\mathrm{b}_{\text {small }}=1.09\right.$ and $b_{\text {large }}=0.74$ ), without affecting our main conclusions. 3: Atta colombica ants. Two (allometric) data points due to diphasic scaling, as in species (2). 4: C. bicolor ants. 5: B. occidentalis bumblebees. 
Drosophila melanogaster [Lanet et al., 2013]. The inverse was true for visually navigating Cataglyphis bicolor ants, where smaller individuals have relatively larger optic lobes [Kühn-Bühlmann and Wehner, 2006]. Our finding that smaller $N$. vitripennis had a similar relative volume of the AL than larger conspecifics was also in line with our expectations, and with the lack of differences in AL cell lineages of smaller D. melanogaster [Lin et al., 2013] and constant relative AL volume in Apis mellifera of different sizes [Gronenberg and Couvillon, 2010]. Direct links between antennal and optic lobe volumes and behavioral patterns have recently been reported for 2 moth species [Stöckl et al., 2016], suggesting that the importance of certain cues may be inferred from relative neuropil size. As mentioned above, $N$. vitripennis primarily uses olfaction rather than vision for host finding [Jacobi, 1939; Whiting, 1967]. This is reflected in our measurements on the developmental plasticity of the antennal and optic lobes: the antennal lobe is of higher importance and does not decrease in relative volume, whereas the optic lobes have fewer functional constraints and could grow to a smaller final relative volume. This confirms that relative neuropil volumes can give important insights into animal behavior.

Of particular interest is the unexpected finding of a relatively smaller mushroom body calyx in small $N$. vitripennis. This structure receives input of the antennal and optic lobes in most hymenopteran species [Gronenberg, 2001]. Part of the reduction in relative calyx volume in smaller individuals may thus have resulted from a reduced input from the smaller optic lobe, although ants did not show a decrease in mushroom body volume after a decrease in the primary sensory neuropils [Waxman et al., 2017]. Vision- or olfaction-specific calyx subunits that are visible in honeybees (i.e., lip and collar) are, however, not distinguishable in N. vitripennis. Therefore, we are unable to attribute this smaller relative calyx volume to changes in modality-specific subunits and must consider calyx volume as a whole. Interestingly, the lateral horn, which is more important for naive odor preferences [Parnas et al., 2013; Strutz et al., 2014], remained constant in relative volume. This suggests that smaller individuals (with a relatively smaller calyx) rely more on naive than on learned behavior. This was indeed demonstrated in a parallel study on the same AsymCx strain of N. vitripennis wasps. Small and large wasps were reared in the same way as in our study, and it was shown that small individuals exhibited reduced visual and olfactory memory performance [van der Woude and Smid, 2017b]. Others have shown that Pieris butterflies with smaller calyces have re- duced learning performance compared to individuals with larger calyces [Snell-Rood et al., 2009]. This finding, however, is in contrast to miniature spiders and T. evanescens, which lack the behavioral consequences of being small [Eberhard, 2011; van der Woude and Smid, 2017b].

The central complex, which for example is important for locomotion and flight [Pfeiffer and Homberg, 2014], was relatively larger in the smallest $N$. vitripennis individuals, which is in line with studies investigating the brains of Bombus impatiens bumblebees [Mares et al., 2005], the honeybee A. mellifera [Gronenberg and Couvillon, 2010], and an interspecific analysis of 3 minute wasps [Makarova and Polilov, 2013]. Unlike the primary visual neuropils, the anterior optic tubercle did not vary in relative volume in our study. Maintaining relative optic tubercle size hints towards a relevance of polarized light [el Jundi et al., 2014] that, to our knowledge, has not been studied for this species.

The neuropil compartments grouped into "rest of the neuropil," which comprised $43-50 \%$ of the total neuropil volume, also had a larger relative volume in small N. vitripennis wasps. Consequences of the large variation in brain and body volume may remain hidden, as this grouping contains a multitude of brain regions that cannot be segmented with the current level of knowledge on the $\mathrm{Na}$ sonia brain. For example, in D. melanogaster, at least 30 more neuropil regions may be specified in the central brain [Ito et al., 2014].

In addition to differences in relative neuropil volumes, the cell body rind is relatively larger in small $N$. vitripennis than in large individuals (Fig. 4). This indicates that the level of volumetric plasticity is lower in cell bodies than in neuropils. Animals of different species, or orders, may use different rules for cellular scaling [HerculanoHouzel et al., 2014]. Depending on the species, changes in total brain volume may affect both cell number or size. The arthropod brain, especially one of this minute size, may pose some unique challenges in uncovering how it responds to the variation we described. For instance, specific neuron subtypes in brains of small and larger T. evanescens do not differ in number, but do differ in size [van der Woude and Smid, 2017a]. For N. vitripennis, more detailed studies are required to ascribe the difference in cell body rind volume to a variation in neuron numbers, neuron/glia ratio, cell body size, or a combination of these factors.

In this study, we used the isogenic AsymCx N. vitripennis line. This allowed for analysis of the phenotypic range of developmental plasticity only, in the absence of genotypic variation. This also implies that our results may 
be genotype specific. Genotype does influence the brainbody size relationship to some extent in isogenic T. evanescens lines; however, all those lines exhibited similar (i.e., isometric) brain-body size relations [van der Woude et al., 2013]. A comparison of body length and head width between the AsymCx line and the outbred HVRx line [van de Zande et al., 2014] (outlined in online suppl. material S1) showed that AsymCx is not an "oddball" regarding its size range; equal variation is found in the N. vitripennis outbred line, as well as in a related species, $N$. giraulti.

\section{Conclusion}

In this study, we provided evidence that brain-body size isometry is not restricted to T. evanescens wasps, but also exists in the small individuals of the slightly larger wasp species $N$. vitripennis. We expect brain-body size isometry to be more common than currently known; it may be present in other minute invertebrates, such as certain species of beetles, springtails, or ants. This trait may be linked to an extreme reduction in arthropod body size beyond a certain threshold, where relative brain volume constitutes too large an energetic cost. This novel and unexpected finding sheds new light on the evolutionary constraints on brains in small bodies, which may be reflected in the changes in neuropil volume, selective adaptation in total cell body, and total neuropil volume, and their effect on cognition, for instance on memory.

\section{Acknowledgements}

We thank E. van der Woude for performing regression analyses and insightful comments, J. Lazebnik for weighing Nasonia wasps, M.E. Huigens for fruitful discussions, M. Dicke and A. Hiscox for help in manuscript preparation, H. Schipper (Wageningen University and Research, Experimental Zoology) for use of the confocal laser scanning microscope, K. Pfeiffer for suggesting AOTu segmentations, and the helpful comments of anonymous reviewers. This work was supported by NWO Open Competition grant 820.01.012.

\section{Disclosure Statement}

The authors have no competing interests to declare.

\section{Author Contributions}

J.G. and H.M.S. designed the study and interpreted the data. J.G. performed experiments, analyzed data, and prepared the manuscript. H.M.S. coordinated the study, performed the procedures described in online supplementary table S3, and helped draft the manuscript.

\section{References}

- Bucher D, Scholz M, Stetter M, Obermayer K, Pflüger HJ (2000): Correction methods for three-dimensional reconstructions from confocal images: I. Tissue shrinking and axial scaling. J Neurosci Methods 100:135-143.

-Bulova S, Purce K, Khodak P, Sulger E, O’Donnell $S$ (2016): Into the black and back: the ecology of brain investment in Neotropical army ants (Formicidae: Dorylinae). Sci Nat 103:1-11.

Charnov EL, Skinner SW (1984): Evolution of host selection and clutch size in parasitoid wasps. Fla Entomol 67:5-21.

Chittka L, Niven J (2009): Are bigger brains better? Curr Biol 19:R995-R1008.

Cobb N (1917): Notes on nemas. Contrib Sci Nematol 5:117-228.

Crawley MJ (2007): Regression; in: The R Book. Chichester, John Wiley \& Sons, pp 387-448.

-Eberhard WG (2011): Are smaller animals behaviourally limited? Lack of clear constraints in miniature spiders. Anim Behav 81:813-823.

Eberhard WG, Wcislo WT (2011): Grade changes in brain-body allometry: morphological and behavioural correlates of brain size in miniature spiders, insects and other inverte- brates; in Casas J (ed): Advances in Insect Physiology. Amsterdam, Elsevier, vol 40, pp 155-214.

el Jundi B, Pfeiffer K, Heinze S, Homberg U (2014): Integration of polarization and chromatic cues in the insect sky compass. J Comp Physiol A 200:575-589.

Gronenberg W (2001): Subdivisions of hymenopteran mushroom body calyces by their afferent supply. J Comp Neurol 435:474-489.

Gronenberg W, Couvillon MJ (2010): Brain composition and olfactory learning in honey bees. Neurobiol Learn Mem 93:435-443.

-Haverkamp A, Smid HM (2014): Octopaminelike immunoreactive neurons in the brain and subesophageal ganglion of the parasitic wasps Nasonia vitripennis and N. giraulti. Cell Tissue Res 358:313-329.

Herculano-Houzel S, Manger PR, Kaas JH (2014): Brain scaling in mammalian evolution as a consequence of concerted and mosaic changes in numbers of neurons and average neuronal cell size. Front Neuroanat 8:77.

Hoedjes KM, Kralemann LEM, van Vugt JJFA, Vet LEM, Smid HM (2014): Unravelling re- ward value: the effect of host value on memory retention in Nasonia parasitic wasps. Anim Behav 96:1-7.

-Hoedjes KM, Steidle JLM, Werren JH, Vet LEM, Smid HM (2012): High-throughput olfactory conditioning and memory retention test show variation in Nasonia parasitic wasps. Genes Brain Behav 11:879-887.

Holm S (1979): A simple sequentially rejective multiple test procedure. Scand J Stat 6:6570.

Ito K, Shinomiya K, Ito M, Armstrong JD, Boyan G, Hartenstein V, Harzsch S, Heisenberg M, Homberg U, Jenett A, Keshishian H, Restifo LL, Rossler W, Simpson JH, Strausfeld NJ, Strauss R, Vosshall LB, Grp IBNW (2014): A systematic nomenclature for the insect brain. Neuron 81:755-765.

Jacobi EF (1939): Über Lebensweise, auffinden des Wirtes und regulierung der individuenzahl von Mormoniella vitripennis Walker. Arch Neer Zool 3:139-282.

-Kilmer JT, Rodríguez RL (2017): Ordinary least squares regression is indicated for studies of allometry. J Evol Biol 30:4-12. 
Kühn-Bühlmann S, Wehner R (2006): Age-dependent and task-related volume changes in the mushroom bodies of visually guided desert ants, Cataglyphis bicolor. J Neurobiol 66: 511-521.

Lanet E, Gould AP, Maurange C (2013): Protection of neuronal diversity at the expense of neuronal numbers during nutrient restriction in the Drosophila visual system. Cell Rep 3: 587-594.

Lin S, Marin EC, Yang CP, Kao CF, Apenteng BA, Huang Y, O'Connor MB, Truman JW, Lee T (2013): Extremes of lineage plasticity in the Drosophila brain. Curr Biol 23:1908-1913.

-Makarova AA, Polilov AA (2013): Peculiarities of the brain organization and fine structure in small insects related to miniaturization. 2. The smallest Hymenoptera (Mymaridae, Trichogrammatidae). Entomol Rev 93:714724.

Mares S, Ash L, Gronenberg W (2005): Brain allometry in bumblebee and honey bee workers. Brain Behav Evol 66:50-61.

Muscedere ML, Traniello JFA (2012): Division of labor in the hyperdiverse ant genus Pheidole is associated with distinct subcaste- and agerelated patterns of worker brain organization. PLoS One 7:e31618.

O'Donnell S, Bulova S (2017): Development and evolution of brain allometry in wasps (Vespidae): size, ecology and sociality. Curr Opin Insect Sci 22:54-61.

O'Donnell S, Clifford MR, DeLeon S, Papa C, Zahedi N, Bulova SJ (2013): Brain size and visual environment predict species differences in paper wasp sensory processing brain regions (Hymenoptera: Vespidae, Polistinae). Brain Behav Evol 82:177-184.

Pagel MD, Harvey PH (1988): The taxon-level problem in the evolution of mammalian brain size - facts and artifacts. Am Nat 132:344359.

Pagel MD, Harvey PH (1989): Taxonomic differences in the scaling of brain on body-weight among mammals. Science 244:1589-1593.

- Parnas M, Lin AC, Huetteroth W, Miesenbock G (2013): Odor discrimination in Drosophila: from neural population codes to behavior. Neuron 79:932-944.

-Pfeiffer K, Homberg U (2014): Organization and functional roles of the central complex in the insect brain. Annu Rev Entomol 59:165-184.
Preibisch S, Saalfeld S, Tomancak P (2009): Globally optimal stitching of tiled 3D microscopic image acquisitions. Bioinformatics 25:14631465.

Rensch B (1948): Histological changes correlated with evolutionary changes of body size. Evolution 2:218-230.

Riveros AJ, Gronenberg W (2010): Brain allometry and neural plasticity in the bumblebee Bombus occidentalis. Brain Behav Evol 75 . 138-148.

Schindelin J, Arganda-Carreras I, Frise E, Kaynig V, Longair M, Pietzsch T, Preibisch S, Rueden C, Saalfeld S, Schmid B, Tinevez JY, White DJ, Hartenstein V, Eliceiri K, Tomancak P, Cardona A (2012): Fiji: an open-source platform for biological-image analysis. Nat Methods 9: 676-682.

Seid MA, Castillo A, Wcislo WT (2011): The allometry of brain miniaturization in ants. Brain Behav Evol 77:5-13.

Smith RJ (2009): Use and misuse of the reduced major axis for line-fitting. Am J Phys Anthropol 140:476-486.

- Smolla M, Ruchty M, Nagel M, Kleineidam CJ (2014): Clearing pigmented insect cuticle to investigate small insects' organs in situ using confocal laser-scanning microscopy (CLSM). Arthropod Struct Dev 43:175-181.

Snell-Rood EC, Papaj DR, Gronenberg W (2009): Brain size: a global or induced cost of learning? Brain Behav Evol 73:111-128.

Stöckl A, Heinze S, Charalabidis A, El Jundi B, Warrant E, Kelber A (2016): Differential investment in visual and olfactory brain areas reflects behavioural choices in hawk moths. Sci Rep 6:26041.

- Strutz A, Soelter J, Baschwitz A, Farhan A, Grabe V, Rybak J, Knaden M, Schmuker M, Hansson BS, Sachse S (2014): Decoding odor quality and intensity in the Drosophila brain. eLife 3:e04147.

Sykes EM, Innocent TM, Pen I, Shuker DM, West SA (2007): Asymmetric larval competition in the parasitoid wasp Nasonia vitripennis: a role in sex allocation? Behav Ecol Sociobiol 61: 1751-1758.

van de Zande L, Ferber S, De Haan A, Beukeboom LW, Van Heerwaarden J, Pannebakker BA (2014): Development of a Nasonia vitripennis outbred laboratory population for genetic analysis. Mol Ecol Res 14:578-587. van der Woude E, Smid HM (2016): How to escape from Haller's rule: olfactory system complexity in small and large Trichogramma evanescens parasitic wasps. J Comp Neurol 524: 1876-1891.

van der Woude E, Smid HM (2017a): Effects of isometric brain-body size scaling on the complexity of monoaminergic neurons in a minute parasitic wasp. Brain Behav Evol 89:185194.

van der Woude E, Smid HM (2017b): Differential effects of brain scaling on memory performance in parasitic wasps. Submitted.

van der Woude E, Smid HM, Chittka L, Huigens ME (2013): Breaking Haller's rule: brain-body size isometry in a minute parasitic wasp. Brain Behav Evol 81:86-92.

-Voje KL, Hansen TF, Egset CK, Bolstad GH, Pélabon C (2014): Allometric constraints and the evolution of allometry. Evolution 68:866885.

Wagh DA, Rasse TM, Asan E, Hofbauer A, Schwenkert I, Durrbeck H, Buchner S, Dabauvalle MC, Schmidt M, Olin G, Wichmann C, Kittel R, Sigrist SJ, Buchner E (2006): Bruchpilot, a protein with homology to ELKS/ CAST, is required for structural integrity and function of synaptic active zones in Drosophila. Neuron 49:833-844.

Warton DI, Duursma RA, Falster DS, Taskinen S (2012): smatr 3 - an R package for estimation and inference about allometric lines. Methods Ecol Evol 3:257-259.

-Waxman HK, Muscedere ML, Traniello JFA (2017): Behavioral performance and neural systems are robust to sensory injury in workers of the ant Pheidole dentata. Brain Beh Evol 89:195-208.

Wehner R, Fukushi T, Isler K (2007): On being small: brain allometry in ants. Brain Behav Evol 69:220-228.

Werren JH, Cohen LB, Gadau J, Ponce R, Baudry E, Lynch JA (2016): Dissection of the complex genetic basis of craniofacial anomalies using haploid genetics and interspecies hybrids in Nasonia wasps. Dev Biol 415:391-405.

Werren JH, Loehlin DW (2009): The parasitoid wasp Nasonia: an emerging model system with haploid male genetics. Cold Spring Harb Protoc 2009:pdb.emo134.

Whiting AR (1967): The biology of the parasitic wasp Mormoniella vitripennis ( $=$ Nasonia brevicornis) (Walker). Q Rev Biol 42:333-406. 